\title{
Skin prick testing a better predictor than blood testing for the diagnosis of peanut allergy in Chinese children
}

\author{
Gilbert T Chua, ${ }^{1}$ Patrick CY Chong, ${ }^{1}$ Elaine YL Au, ${ }^{2}$ KN Cheong, ${ }^{1}$ Wilfred HS Wong, ${ }^{1}$ \\ Eric YT Chan, ${ }^{2}$ Marco HK Ho, ${ }^{1}$ YL Lau, ${ }^{1}$ Jaime S Rosa Duque ${ }^{1}$
}

\begin{abstract}
Background: Peanut allergy is common in Chinese children, yet the most predictive diagnostic cut-offs for skin prick test (SPT) and blood testing in this population are unclear.

Objectives: We aimed to determine the optimal cut-off values for whole-peanut SPT, specific IgE (sIgE) and component-resolved diagnostics (CRD) for Chinese children based on outcomes of open oral food challenges (OFC) to peanut.
\end{abstract}

Methods: We recruited ethnic-Chinese patients 1-18 years old who were suspected of having peanut allergy based on a history of reactions after exposure or sensitization although peanut naïve. Considering the AUC value of $0.8,80 \%$ power and 5\% level of significance with two tails, 26 patients were needed. Sensitivities, specificities, positive and negative predictive values, and receiver operating characteristic curves (ROCs) and their area-under-curves (AUCs) for SPT, peanut sIgE, and CRD were compared.

Results: Thirty-one subjects participated. Only SPT reached statistical significance (AUC 0.91, $p=0.0001$ ), but not the other tests. Seven retrospective data were added to optimize the power. SPT remained to be the best predictor, followed by Ara h $2 \operatorname{sigE}(A U C 0.72, p=0.02$ ). An SPT wheal size of $3 \mathrm{~mm}$ and Ara h 2 sIgE of $0.14 \mathrm{kU}(\mathrm{A}) / \mathrm{L}$ yielded the highest Youden's index. The specificity of SPT and Ara h 2 sIgE reached $94 \%$ at $6 \mathrm{~mm}$ and $0.74 \mathrm{kU}(\mathrm{A}) / \mathrm{L}$, respectively. Comparisons of ROCs revealed that SPT was significantly better than Ara h $2 \operatorname{sigE}(p=0.03)$ and whole-peanut sIgE (AUC $0.61, p=0.26)$.

Conclusion: In Chinese children, SPT appeared to be the best predictor for peanut allergy, followed by Ara h 2 sIgE.

Key words: Peanut Allergy, Chinese, Children, Skin Prick Test, Component Resolved Diagnostics

\footnotetext{
Affiliations:

Department of Paediatrics and Adolescent Medicine, Queen Mary Hospital, Li Ka Shing Faculty of Medicine, The University of Hong Kong, Hong Kong, China

2 Division of Clinical Immunology, Department of Pathology

\& Clinical Biochemistry, Queen Mary Hospital, Hong Kong, China
}

\author{
Corresponding author: \\ Jaime S Rosa Duque \\ Room 115, New Clinical Building, Queen Mary Hospital, \\ 102 Pokfulam Road, Hong Kong \\ E-mail: jsrduque@hku.hk
}

\section{Introduction}

The prevalence of peanut allergy in the Chinese population is high and estimated to be $0.3-0.7 \% .^{1-4}$ Food allergies significantly impact the quality of life of patients and their families. ${ }^{5,6}$ Patients with food allergies experienced depressed moods, felt anxious about food safety and social occasions, and had to bear more expensive food costs. ${ }^{7}$ Therefore, providing an accurate diagnosis is crucial. This process relies on the acquisition of relevant clinical histories and reliable investigations, including skin prick test (SPT) and blood tests.
Some patients may ultimately require supervised food challenges for confirmation. However, as evident from the tragic event involving a three-year-old child who died due to a routine oral food challenge, performing tests that carry less risks with high pre-test probabilities prior food challenges is paramount. $^{8}$

Generally, patients with convincing histories are considered as peanut allergic if testing reveals large wheal sizes on skin prick testing (SPT) or high specific IgE (sIgE) levels 
to peanut. A wheal diameter $\geq 8 \mathrm{~mm}$ for children older than 2 years old has been shown to yield a positive predictive value (PPV) of $96-100 \%$, and a wheal diameter $\geq 4 \mathrm{~mm}$ for children younger than 2 years old yielded a PPV of $100 \% .{ }^{9,10}$ For blood testing, PPV of $98 \%$ and $95 \%$ have been reported for values of $15 \mathrm{kUA} / \mathrm{L}$ for peanut $\operatorname{sigE}$ and $0.1 \mathrm{kUA} / \mathrm{L}$ for Ara h 2 sIgE, respectively. ${ }^{9,11}$ However, investigators from different countries have reported variable cut-off values for SPT, peanut sIgE and CRD. For SPT, Sicherer and Wood reviewed studies from Australia, ${ }^{10,12}$ France, ${ }^{13}$ Canada, ${ }^{14}$ US, ${ }^{15}$ and UK ${ }^{16}$ which demonstrated different cut-offs that achieved 100\% PPV, ranging from $4 \mathrm{~mm}$ in younger age groups to up to $16 \mathrm{~mm}$ in older children. Additionally, these studies varied in terms of age of the subjects, challenge criteria and skin test reagents used. ${ }^{17}$ As for blood tests, such as the component-resolved diagnostics (CRD) which is becoming used more widely, their sensitivities, specificities, PPV and NPV reported have been inconsistent depending on which region of the world the study was performed. For example, peanut sIgE at $10 \mathrm{kU} / \mathrm{L}$ and Ara $\mathrm{h} 2$ SIgE at $4.0 \mathrm{kU} / \mathrm{L}$ achieved $100 \% \mathrm{PPV}$ in Korea. ${ }^{18}$ In contrast, Ara h 2 sIgE reached the sensitivity and specificity of $88 \%$ and $84 \%$, respectively, at a cut-off of $0.35 \mathrm{kU}(\mathrm{A}) / \mathrm{L}$ for Japanese children between the ages of 2 and 13 years old. ${ }^{19}$ A recent study conducted in Australia by the HealthNuts Study group showed cut-off values of $14.9 \mathrm{kU} / \mathrm{L}$ for peanut sIgE and 1.19 $\mathrm{kU} / \mathrm{L}$ for Ara h 2 sIgE that achieved the specificity of $98 \%{ }^{11}$ Two other conflicting studies, one in Sweden and another in the US, showed that Ara h 2 of $0.35 \mathrm{kU} / \mathrm{L}$ had a 93\% predictive value. ${ }^{20}$

Moreover, sensitization patterns seem to differ even within the same country and ethnic group. For example, a study in Beijing, China demonstrated that Ara h 9 was the major peanut allergen and mono-sensitization to this component was the most common pattern for their population, ${ }^{21}$ while in Taiwan, Ara h 2 was the most important component associated with peanut allergy. ${ }^{22}$ Nevertheless, whether these patients actually had peanut allergy remains nebulous as both studies used questionnaires rather than the confirmatory gold-standard diagnostic testing modality for the diagnosis of food allergy: the oral food challenge (OFC).

The most likely reason for these inconsistent conclusions is that skin and laboratory testing for different ethnicities and regions of the world may be different. As such, a set of ethnic -specific cut-off values for predicting peanut allergy is needed. This study aims to determine and compare the utility of peanut SPT, peanut sIgE and CRDs for Chinese children based on oral food challenge outcomes.

\section{Materials and Methods}

This was a single-center, cross-sectional study. The inclusion criteria were as follows: patients between the ages of 1 to 18 years old who were of ethnic-Chinese descent suspected of having peanut allergy based on a history of reactions after exposure to peanut or sensitization although peanut naive. $\mathrm{Pa}-$ tients were recruited from the Allergy Clinic at the Department of Paediatrics and Adolescent Medicine, Queen Mary Hospital. A detailed clinical history was recorded and those who fulfilled the aforementioned criteria were invited to participate.
Prior to entry into the study, parents and guardians were thoroughly counselled on the potential risks and benefits before they signed informed consent forms approved by the Institutional Review Board of the University of Hong Kong/ Hospital Authority Hong Kong West Cluster (IRB reference number: UW 17-033). SPT and blood tests for peanut sIgE and CRD (Ara h 1, 2, 3, 8 and 9 sIgE) were obtained. All participants underwent an in-patient open peanut challenge. A physician and registered nurse were available at all times during the challenge procedure. In the event of any allergic symptoms, the doctor-in-charge was immediately informed to conduct an assessment. Standby rescue medications were readily available as needed.

\section{Skin Prick Testing}

SPT was performed using single-head lancets on the forearms of children with histamine $(1 \mathrm{mg} / \mathrm{ml})$ as positive control, glycerinated saline as negative control and whole-peanut extract (ALK- Abelló, Denmark). Wheal and flare sizes were measured after 15 minutes. Patients abstained from anti-histamine-containing medications and topical corticosteroids on their forearms for at least one week prior to the procedure.

\section{Peanut sIgE and Component Resolved Diagnostics}

Three to $5 \mathrm{ml}$ of clotted blood were drawn and sent to the Queen Mary Hospital Immunology Laboratory. Serum was extracted for quantification of peanut sIgE and CRD (Ara $h$ $1,2,3,8,9)$ by ImmunoCAP (Thermo Fisher Scientific Inc, Massachusettes, USA).

\section{Peanut Challenge}

The OFCs to peanut were performed according to the Australian Society of Clinical Immunology and Allergy (ASCIA) peanut challenge protocol. ${ }^{23}$ We used a commercial smooth peanut butter preparation (Skippy ${ }^{\oplus}$, Hormel Foods Corporation, Minnesota, USA). One teaspoon $(5 \mathrm{ml})$ of peanut butter was deemed equivalent to 3.5 grams of peanut protein (Product information).

The OFC was performed as an in-patient procedure, starting with a smear of peanut butter applied to the buccal mucosa of the lower lip. Subsequently, at 20-minute intervals, doses of $1 / 8,1 / 4,1 / 2$ and 1 teaspoon of peanut butter were given. If no reaction occurred during the entire period, the participant was deemed peanut tolerant. Those who developed acute allergic reactions, such as urticaria, angioedema, vomiting, wheezing, hypoxia, hypotension, and anaphylaxis during any stage of the challenge were diagnosed as peanut allergic. A repeat open OFC was performed for equivocal results.

\section{Statistical Analysis}

\section{Power Analysis}

We considered the ratio between the peanut tolerant and allergic groups to be $1: 1$ and the AUC value to be 0.8 . To achieve an $80 \%$ power at $5 \%$ level of significance with two tails, a total of 26 subjects was needed. For an AUC value of 0.75 , a total of 38 subjects would be needed. 


\section{Data Analysis}

Power analysis was performed using $\mathrm{G}^{\star}$ Power (Heinrich-Heine-University Düsseldorf, Düsseldorf, Germany). Data were analysed using Microsoft Excel $^{\oplus}$ (Redmond, Washington, USA) and GraphPad ${ }^{\circledast}$ Prism 8 (San Diego, California, USA). For comparison of data between the tolerant and allergic groups, the Fisher's exact test was used for categorical variables, whereas the Mann-Whitney $U$ test was used for continuous variables.

Receiver-operating characteristic curves (ROC) were generated by plotting sensitivities against 1 -specificities. The area-under-curve (AUC), Youden's Index, and p-value of each test were calculated. A Youden's Index closest to 1 indicated the test's value with the highest sensitivity and specificity. Comparison of ROC curves was performed using the Hanley and McNeil Method. A $p$-value of $<0.05$ was treated as statistically significant.

\section{Results}

Between January 2017 and September 2018, we prospectively recruited 64 patients who were suspected of having peanut allergy based on a history of reactions after exposure to peanut or sensitization although peanut naïve. Thirty-one patients and parents did not wish to proceed to OFC due to its inconvenience or they had serious concerns over potential severe allergic reactions. Two patients were also excluded due to self-reported reactions after accidental exposure to peanut and therefore declined the OFC.
A total of 31 ethnic-Chinese subjects participated and their characteristics are listed in Table 1. The overall median age of this cohort was 4.54 years old, and $83.9 \%$ were older than 2 years old. Sixteen patients $(51.6 \%)$ were reported to be peanut-naïve at the time of challenge. Sixteen patients $(51.6 \%)$ had positive challenge results. No patient in this entire study developed severe reactions during the OFC.

When comparing between the peanut allergic and tolerant groups, there were no differences in their demographic data. The median SPT wheal size diameters $(6 \mathrm{~mm}$ vs $1 \mathrm{~mm}$, $p<0.0001)$ and Ara h $2 \operatorname{sigE}(0.28$ vs $0.1 \mathrm{kU}(\mathrm{A}) / \mathrm{L}, p=0.04)$ were significantly higher in the peanut-allergic group than the tolerant group. Conversely, the tolerant group had significantly higher Ara h 8 sIgE than the allergic group ( 0.18 vs $0.1 \mathrm{kU}(\mathrm{A}) / \mathrm{L}, p=0.04$ ) (Figure 1). There were no differences in peanut sIgE, Ara h 1, 3, and 9 sIgE levels between groups. ROC curve of SPT achieved statistical significance with AUC $0.91(p=0.0001)$ but not for Ara h 2 sIgE (AUC 0.7, $p=$ 0.053 ) and peanut sIgE (AUC 0.6, $p=0.32$ ).

Since the AUC and p value for Ara h 2 sIgE were close but did not reach significance possibly due to inadequate power, we subsequently included seven additional sets of retrospective data based on the power calculation that a sample size of 38 subjects was needed for a target AUC value of 0.75 . Retrospective data were used due to limitation of funding and nursing care for more prospective OFCs and our past procedures followed an identical protocol and approach. Patients who received SPT, sIgE and CRD measurements, and OFCs

Table 1. Patients demographics

\begin{tabular}{|c|c|c|c|c|}
\hline & Overall $(n=31)$ & Allergic $(n=16)$ & Tolerant $(n=15)$ & $p$ value \\
\hline Male Gender (\%) & $20(65)$ & $11(69)$ & $9(60)$ & 0.72 \\
\hline Age (years) & $4.54(3.65-6.46)$ & $4.12(2.21-6.48)$ & $4.68(3.87-8.25)$ & 0.32 \\
\hline Peanut Naïve (\%) & $16(52)$ & $7(44)$ & $9(60)$ & 0.48 \\
\hline Eczema (\%) & $20(65)$ & $10(63)$ & $10(67)$ & 0.99 \\
\hline Asthma (\%) & $6(19)$ & $4(25)$ & $2(13)$ & 0.65 \\
\hline Allergic Rhinitis (\%) & $11(35)$ & $6(38)$ & $5(33)$ & 0.99 \\
\hline SPT $(\mathrm{mm})$ & $3(1-4)$ & $6(3-10)$ & $1(0-2)$ & $<0.0001^{\star}$ \\
\hline Peanut sIgE $[\mathrm{kU}(\mathrm{A}) / \mathrm{L}]$ & $1.11(0.20-2.64)$ & $1.39(0.22-3.41)$ & $0.26(0.1-4.74)$ & 0.33 \\
\hline Ara h 1 sIgE $[\mathrm{kU}(\mathrm{A}) / \mathrm{L}]$ & $0.12(0.10-0.29)$ & $0.17(0.10-0.72)$ & $0.10(0.10-0.47)$ & 0.17 \\
\hline Ara h $2 \operatorname{sigE}[k U(A) / L]$ & $0.18(0.10-0.37)$ & $0.28(0.10-0.88)$ & $0.10(0.10-0.23)$ & $0.04^{*}$ \\
\hline Ara h $3 \operatorname{sigE}[k U(A) / L]$ & $0.10(0.10-0.19)$ & $0.10(0.10-0.19)$ & $0.10(0.10-0.59)$ & 0.33 \\
\hline Ara h $8 \operatorname{sigE}[k U(A) / L]$ & 0.10 & 0.10 & $0.18(0.10-0.25)$ & $0.04^{*}$ \\
\hline Ara h $9 \operatorname{sigE}[k U(A) / L]$ & 0.10 & 0.10 & $0.10(0.10-0.11)$ & 0.27 \\
\hline
\end{tabular}

$\mathrm{SPT}=$ skin prick test, $\operatorname{sIgE}=$ specific immunoglobulin $\mathrm{E}$

Data are median values (and 95\% interpercentiles or $95 \%$ confidence intervals). ${ }^{\star}=p<0.05$ 


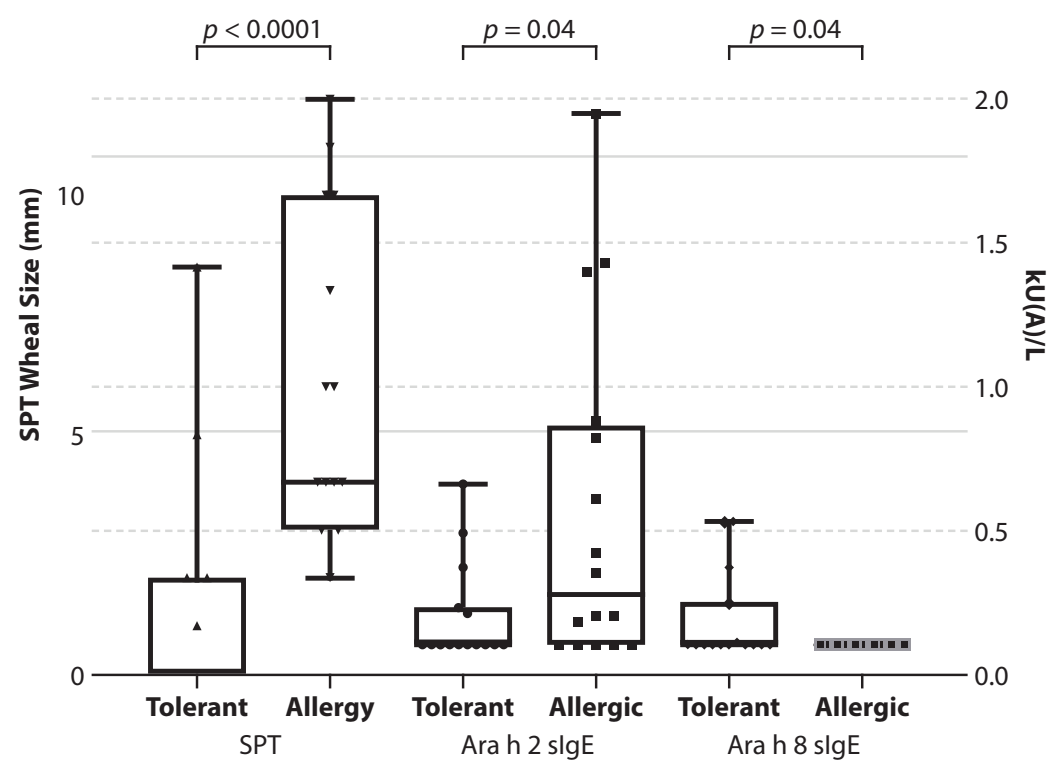

Figure 1. Box-and-whisker diagrams for skin prick tests (SPT), Ara h 2 and 8 specific immunoglobulin E (sIgEs). The peanut-allergic group had significantly greater SPT wheal diameters, Ara h 2 sIgE and Ara h 8 sIgE compared to the peanut-tolerant group.

to peanut according to our same clinical protocol as described above prior to the commencement of this study were included for analysis. These patients fulfilled the inclusion and exclusion criteria of this study. Their median age was 6.39 years. Five of them were boys (71.4\%). The median SPT wheal size, peanut sIgE and Ara h 2 sIgE were $5 \mathrm{~mm}, 1.99 \mathrm{kU}(\mathrm{A}) / \mathrm{L}$ and $0.62 \mathrm{kU}(\mathrm{A}) / \mathrm{L}$ respectively. Five $(71.4 \%)$ were challenge positive. All had mild reactions, such as urticaria and throat itchiness. None had anaphylaxis.

(a)

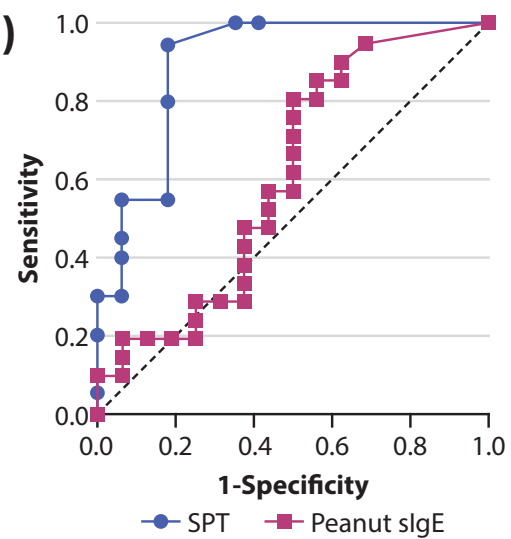

For these 38 total patients, both ROC curves of SPT and Ara h 2 sIgE achieved statistical significance (Figure 2a and 2b) with AUC's of $0.9(p<0.0001)$ and $0.72(p=0.02)$, respectively. Sensitivities, specificities, positive and negative predictive values, and Youden's indices of SPT wheal size and Ara h 2 sIgE are listed in Tables 2 and 3, respectively. SPT wheal diameter of $3 \mathrm{~mm}$ achieved the highest Youden's index at 0.77 , which yielded a sensitivity of $95 \%$ and specificity of $82 \%$.

(b)

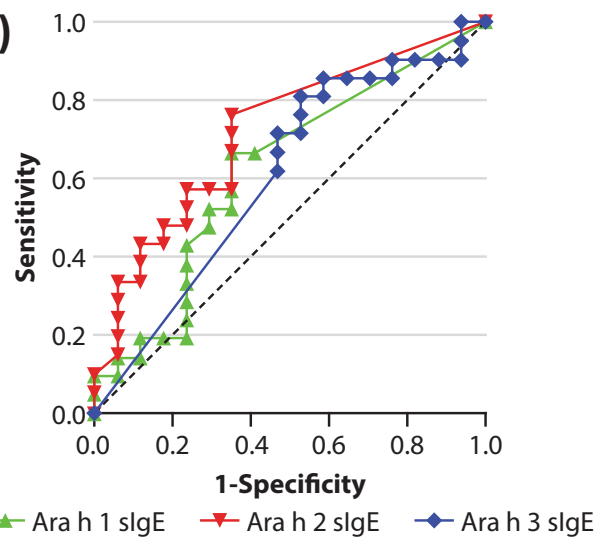

Figure 2. Receiver operative characteristics (ROC) analysis indicated that skin prick tests (SPT) but not peanut-specific IgE (sIgE) had a significant association with peanut allergy (AUC $0.90, p<0.0001$ and AUC $0.61, p=0.26$, respectively) (Figure 2a). ROC analysis of peanut component-resolved diagnostics (CRD) revealed only Ara h 2 sIgE (AUC 0.72, $p=0.02)$ had significant association with peanut allergy, while Ara h 1 sIgE (AUC 0.62, $p=0.19$ ) and Ara h $3 \operatorname{sigE}($ AUC 0.60, $p=0.27$ ) did not (Figure $2 \mathrm{~b}$ ). Comparison of ROC curves between SPT and Ara $h 2$ sIgE demonstrated that SPT had a significantly greater AUC $(p=0.03$ ) (Figure $2 c)$. Children older than 2 years old (AUC $0.96, p<0.0001$ ) had a greater AUC than the younger group (AUC $0.75, p=0.29$ ), although the difference did not reach significance (Comparison of ROC curves $p=$ 0.29) (Figure 2d). 
(c)

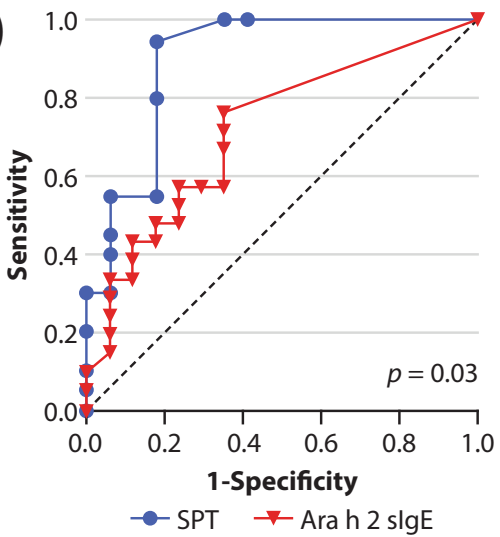

(d)

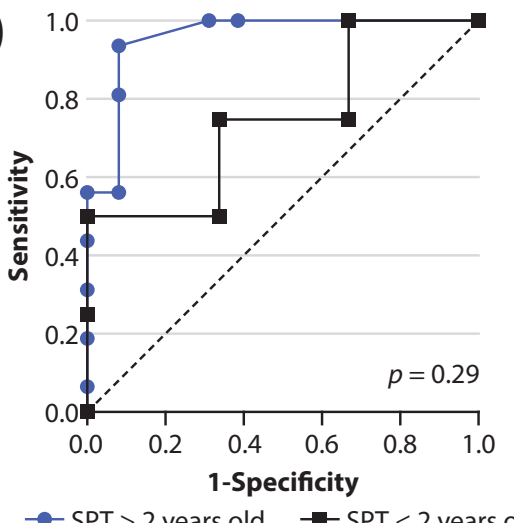

Figure 2. (Continued)

Table 2. Sensitivity, specificity, PPV, NPV and Youden's index of various cut-offs for SPT wheal size

\begin{tabular}{cccccc}
$\begin{array}{c}\text { SPT wheal } \\
\text { size }(\mathbf{m m})\end{array}$ & Sensitivity & Specificity & PPV & NPV & $\begin{array}{c}\text { Youden's } \\
\text { Index }\end{array}$ \\
\hline$\geq 2$ & 1 & 0.65 & 0.77 & 1.00 & 0.65 \\
$\geq 3$ & 0.95 & 0.82 & 0.86 & 0.93 & $0.77^{*}$ \\
$\geq 4$ & 0.8 & 0.82 & 0.84 & 0.78 & 0.62 \\
$\geq 5$ & 0.55 & 0.82 & 0.78 & 0.61 & 0.37 \\
$\geq 6$ & 0.55 & 0.94 & 0.91 & 0.64 & 0.49 \\
$\geq 7$ & 0.45 & 0.94 & 0.90 & 0.59 & 0.39 \\
$\geq 8$ & 0.4 & 0.94 & 0.89 & 0.57 & 0.34 \\
$\geq 9$ & 0.3 & 1 & 1.00 & 0.55 & 0.3 \\
\hline
\end{tabular}

* $=$ highest Youden's index

$\mathrm{SPT}=$ skin prick test $\mathrm{PPV}=$ positive predictive value $\mathrm{NPV}=$ negative predictive value

Table 3. Sensitivity, specificity, PPV, NPV and Youden's index of various cut-offs for Ara h 2 sIgE

\begin{tabular}{cccccc}
$\begin{array}{c}\text { Ara h } 2 \text { sIgE } \\
{[\mathbf{k U}(\mathbf{A}) / \mathrm{L}]}\end{array}$ & Sensitivity & Specificity & PPV & NPV & $\begin{array}{c}\text { Youden's } \\
\text { Index }\end{array}$ \\
\hline$\geq 0.14$ & 0.76 & 0.65 & 0.72 & 0.70 & $0.41^{\star}$ \\
$\geq 0.18$ & 0.71 & 0.65 & 0.70 & 0.66 & 0.36 \\
$\geq 0.19$ & 0.67 & 0.65 & 0.69 & 0.63 & 0.32 \\
$\geq 0.21$ & 0.57 & 0.65 & 0.66 & 0.56 & 0.22 \\
$\geq 0.22$ & 0.57 & 0.71 & 0.70 & 0.58 & 0.28 \\
$\geq 0.27$ & 0.57 & 0.76 & 0.74 & 0.60 & 0.33 \\
$\geq 0.33$ & 0.52 & 0.76 & 0.72 & 0.57 & 0.28 \\
$\geq 0.36$ & 0.48 & 0.76 & 0.70 & 0.55 & 0.24 \\
$\geq 0.40$ & 0.48 & 0.82 & 0.76 & 0.57 & 0.3 \\
$\geq 0.46$ & 0.43 & 0.82 & 0.74 & 0.55 & 0.25 \\
$\geq 0.55$ & 0.43 & 0.88 & 0.81 & 0.57 & 0.31 \\
$\geq 0.62$ & 0.38 & 0.88 & 0.79 & 0.55 & 0.26 \\
$\geq 0.64$ & 0.33 & 0.88 & 0.76 & 0.53 & 0.21 \\
$\geq 0.74$ & 0.33 & 0.94 & 0.87 & 0.54 & 0.27 \\
\hline & & & & &
\end{tabular}


Table 3. (Continued)

\begin{tabular}{cccccc}
$\begin{array}{c}\text { Ara h 2 sIgE } \\
{[\mathrm{kU}(\mathrm{A}) / \mathrm{L}]}\end{array}$ & Sensitivity & Specificity & PPV & NPV & $\begin{array}{c}\text { Youden's } \\
\text { Index }\end{array}$ \\
$\mathbf{2}$ & 0.85 & 0.94 & 0.85 & 0.53 & 0.23 \\
$\geq 0.95$ & 0.24 & 0.94 & 0.82 & 0.51 & 0.18 \\
$\geq 1.2$ & 0.19 & 0.94 & 0.79 & 0.50 & 0.13 \\
$\geq 1.4$ & 0.14 & 0.94 & 0.73 & 0.48 & 0.08 \\
$\geq 1.7$ & 0.095 & 1 & 1.00 & 0.48 & 0.095 \\
$\geq 8.9$ & 0.048 & 1 & 1.00 & 0.47 & 0.048 \\
\hline
\end{tabular}

* $=$ highest Youden's index

sIgE $=$ specific immunoglobulin $\mathrm{E}, \mathrm{PPV}=$ positive predictive values, NPV = negative predictive values

The specificity of SPT reached $\sim 95 \%(94 \%)$ at $6 \mathrm{~mm}$. Ara h 2 sIgE of $0.14 \mathrm{kU}(\mathrm{A}) / \mathrm{L}$ achieved the highest Youden's index at 0.41 , with a corresponding sensitivity of $76 \%$ and specificity of $65 \%$. The $~ 95 \%$ (94\%) specificity for Ara h 2 sIgE was at $0.74 \mathrm{kU}(\mathrm{A}) / \mathrm{L}$. The AUC of peanut $\operatorname{sigE}$ was 0.61 , which was not statistically significance $(p=0.26)$. The ROC curve of SPT was significantly better than Ara h $2 \operatorname{sigE}(p=0.03)$ (Figure 2c). The Pearson correlation coefficients (r2) for SPT, peanut sIgE and CRD's against the threshold doses amongst challenge positive patient were all $<0.5$.

In addition, we compared patients who were older versus younger than 2 years old, as a previous study had demonstrated important differences in cut-off values for these 2 different age groups. ${ }^{9}$ For patients older than 2 years old, the AUC of SPT was $0.96(p<0.0001)$, with the wheal size for the highest sensitivity and specificity also at $3 \mathrm{~mm}$, both achieving $90 \%$ (Figure 2d); for patients younger than 2 years old, the AUC was 0.75 but did not reach statistical significance $(p=0.29)$.

For the 12 patients whose SPT wheal size diameters were between 3 and $6 \mathrm{~mm}, 6$ had Ara h $2 \operatorname{sigE}<0.1 \mathrm{kU}(\mathrm{A}) / \mathrm{L}$ and 2 of them were tolerant to peanut.

\section{Discussion}

To our knowledge, the present study provides the only set of sensitivity and specificity CRD data for Chinese peanut-allergic patients based on the gold standard OFC test. Time between recruitment and entry to food challenge was reasonable, with the median time of 3.63 months. Our study demonstrated that, among Chinese children, SPT yielded the highest sensitivity and specificity at a wheal size of $3 \mathrm{~mm}$, while a wheal size of $6 \mathrm{~mm}$ provided a $\sim 95 \%$ specificity. For Ara h 2 sIgE, the highest sensitivity and specificity was achieved at $0.14 \mathrm{kU}(\mathrm{A}) / \mathrm{L}$ and the $\sim 95 \%$ specificity was $0.74 \mathrm{kU}(\mathrm{A}) / \mathrm{L}$. Overall, the best predictor for the diagnosis of peanut allergy based on OFC was SPT, while Ara h 2 sIgE was the best predictor amongst all blood tests.

Practically, SPT is a convenient test that is generally more widely available than the more novel allergen-specific CRD. Results for SPT can be obtained within 15 minutes, while final reports of CRD may require several days of waiting time. Almost all allergy centres across the world utilize the SPT since it is a convenient and rapid diagnostic tool for the workup of food allergies. The cut-off value obtained from this study

Table 4. Studies comparing the performances of SPT, peanut sIgE and Ara h 2 sIgE

\begin{tabular}{|c|c|c|c|c|c|c|c|c|}
\hline \multirow{2}{*}{ References } & \multirow{2}{*}{ Country } & \multirow{2}{*}{ Methods } & \multicolumn{2}{|c|}{ SPT } & \multicolumn{2}{|c|}{ Peanut sIgE } & \multicolumn{2}{|c|}{ Ara h 2 sIgE } \\
\hline & & & AUC & p-value & AUC & p-value & AUC & p-value \\
\hline Li $2018^{24}$ & United Kingdom & OFC & 0.95 & $<0.01$ & 0.489 & 0.26 & 0.779 & $<0.01$ \\
\hline Simms $2017^{25}$ & Canada & OFC & 0.93 & $<0.0001$ & 0.812 & 0.008 & N/A & N/A \\
\hline Gray 201526 & South Africa & $\begin{array}{l}\text { Questionnaire } \\
\text { (OFC if uncertain) }\end{array}$ & $0.88-1.00$ & $\mathrm{~N} / \mathrm{A}$ & $0.67-0.72$ & $\mathrm{~N} / \mathrm{A}$ & $0.81-0.87$ & N/A \\
\hline Suratannon $2013^{27}$ & Thailand & $\begin{array}{c}\text { OFC } \\
\text { (DBPCFC if subjective } \\
\text { symptoms) }\end{array}$ & 0.62 & N/A & 0.51 & N/A & 0.82 & N/A \\
\hline $\begin{array}{l}\text { Chua } 2019 \\
\text { (Current study) }\end{array}$ & Hong Kong, China & OFC & 0.9 & $<0.0001$ & 0.61 & 0.26 & 0.72 & 0.02 \\
\hline
\end{tabular}

$\mathrm{OFC}=$ oral food challenge, $\mathrm{DBPCFC}=$ double-blind, placebo-controlled food challenge, $\mathrm{SPT}=$ skin prick tests, $\mathrm{sIgE}=$ specific immunoglobulin $\mathrm{E}, \mathrm{N} / \mathrm{A}=$ not available 
suggests that patients with wheal size $\geq 6 \mathrm{~mm}$, rather than 8 $\mathrm{mm}$ based on studies such as those conducted in Australia, ${ }^{11}$ has a high likelihood of peanut allergy for Chinese children. As such, proceeding to OFC for these patients could carry a significant risk of allergic reaction and should be avoided or considered with caution depending on the clinical history.

To date, there has only been a few studies that performed direct comparisons between SPT and CRD using OFC outcomes, and no data were available in Chinese children or in this region of the world. In this study, SPT was found to be the best predictor and more superior to Ara $\mathrm{h} 2$ and peanut sIgE for the diagnosis of peanut allergy, and these results are congruent to studies conducted in the UK, Canada and South Africa but not Thailand (Table 4). ${ }^{24-27}$

A proportion of patients, especially amongst the tolerant group, were Ara h 8 sensitized, with the median value being higher in the tolerant group than the allergic group. The peanut profilin component, Ara h 8, shares birch pollen cross sensitization due to its homology with Bet $\mathrm{v} 1$, and a recent study demonstrated that isolated Ara h 8 sensitization is a strong indicator for peanut tolerance. ${ }^{28}$ These observations are consistent with findings from this study. However, birch trees are rare in this locality of Hong Kong, and therefore we surmise that sensitization to birch pollen components occurred due to our patients' travels to other areas of the world. Indeed, other regions of China, Asia, and the world contains high airborne levels of birch pollens. ${ }^{29}$ More studies carefully interrogating a thorough travel history and associated allergic rhinitis symptoms, and possibly nasal challenges to birch pollens and Ara h 8 will be needed to understand the full implication of this profilin component for peanut allergy in this region.

The ultimate gold standard, the double blind, placebo-controlled food challenge test (DBPCFC), was not employed in this study. However, OFC is a reasonable and practical alternative to a complex and labour-intensive DBPCFC when time and resources are limited. ${ }^{30}$ In addition, all our patients who were challenge positive were confirmed by observing objective symptoms, such as visible urticaria or lips swelling, but not subjective symptoms alone such as throat itchiness. Secondly, our study had a relatively small sample size for children younger than two years old. Therefore, there was inadequate power to determine a reliable cut-off for this age group and whether there are significant differences compared to older children. Third, some analyses for a few subjects were obtained retrospectively, although heterogeneity is likely minimal as patients included in the analysis fulfilled the same inclusion and exclusion criteria. Fourth, there was also potential referral bias and therefore these findings may not be representative of the entire population of peanut-allergic children. On the other hand, findings from this study may be applicable for Chinese children who are referred to tertiary allergy centers. Some patients did not participate due to inconvenience or high SPT or blood testing results, which may have contributed to selection bias. However, safety and ethical consideration are of utmost importance and patients who have valid concerns over the possibility of anaphylactic death must be respected. ${ }^{8}$

\section{Conclusion}

Using the gold-standard OFC, our study demonstrated that SPT was the best predictor for peanut allergy among Chinese children and was superior to sIgE and CRD. There is a high likelihood that patients with peanut SPT wheal diameter size $>6 \mathrm{~mm}$ are peanut allergic. CRD testing for Ara h 2 and possibly Ara h 8 may be a reasonable alternative if patients are unable to undergo SPT, such as those who are uncooperative, at risk of reaction due to skin testing, unable to tolerate interruption of their anti-histamines or immunosuppressants, or have inadequate skin reactivity. Further studies are warranted to determine the most predictive testing values among children younger than 2 years old.

\section{Acknowledgement}

The study team would like to thank all personnel who assisted with the procedures, clinicians who referred patients to us for this study, and our patients and families for their participation. This study is supported by the Hong Kong Institute of Allergy Research Grant.

\section{References}

1. Leung TF, Yung E, Wong YS, Lam CW, Wong GW. Parent-reported adverse food reactions in Hong Kong Chinese pre-schoolers: epidemiology, clinical spectrum and risk factors. Pediatr Allergy Immunol. 2009;20(4):339-46.

2. Wu TC, Tsai TC, Huang CF, Chang FY, Lin CC, Huang IF et al. Prevalence of food allergy in Taiwan: a questionnaire-based survey. Intern Med J. 2012;42(12):1310-5.

3. Chen J, Hu Y, Allen KJ, Ho MH, Li H. The prevalence of food allergy in infants in Chongqing, China. Pediatr Allergy Immunol. 2011;22:356-60.

4. Zeng GQ, Luo JY, Huang HM, Zheng PY, Luo WT, Wei NL, et al. Food allergy and related risk factors in 2540 preschool children: an epidemiological survey in Guangdong Province, Southern China. World J Pediatr. 2015;11:219-25.

5. Walkner M, Warren C, Gupta RS. Quality of Life in Food Allergy Patients and Their Families. Pediatr Clin North Am. 2015 Dec;62(6):1453-61.

6. Peniamina RL, Mirosa M, Bremer P, Conner TS. The stress of food allergy issues in daily life. Psychol Health. 2016;31(6):750-67.

7. DunnGalvin A, Dubois AE, Flokstra-de Blok BM, Hourihane JO. The effects of food allergy on quality of life. Chem Immunol Allergy. 2015;101:235-52.

8. American College of Allergy, Asthma \& Immunology [Internet]. Illinois: Allergists Responded to Death of 3 year-old Boy During Oral Food Challenge. Statement by the American College of Allergy, Asthma \& Immunology; American Academy of Allergy, Asthma \& Immunology; and the Canadian Society of Allergy and Clinical Immunology; c2017 [cited 2019 Mar 13]. Available from: https://acaai.org/allergists-respond-death-3 -year-old-boy-during-oral-food-challenge

9. Hill DJ, Heine RG, Hosking CS. The diagnostic value of skin prick testing in children with food allergy. Pediatr Allergy Immunol. 2004:15:435-41.

10. Ho $\mathrm{MH}$, Heine RG, Wong W, Hill DJ. Diagnostic accuracy of skin prick testing in children with tree nut allergy. J Allergy Clin Immunol. 2006;117(6):1506-8.

11. Dang TD, Tang M, Choo S, Licciardi PV, Koplin JJ, Martin PE, et al. Increasing the accuracy of peanut allergy diagnosis by using Ara h 2 . J Allergy Clin Immunol. 2012;129(4):1056-63.

12. Sporik R, Hill DJ, Hosking CS. Specificity of allergen skin testing in predicting positive open food challenges to milk, egg and peanut in children. Clin Exp Allergy. 2000;30:1541-6.

13. Rancé F, Abbal M, Lauwers-Cancès V. Improved screening for peanut allergy by the combined use of skin prick tests and specific IgE assays. J Allergy Clin Immunol. 2002;109:1027-33.

14. Pucar F, Kagan R, Lim H, Clarke AE. Peanut challenge: a retrospective study of 140 patients. Clin Exp Allergy. 2001;31:40-6.

15. Sampson HA, Ho DG. Relationship between food-specific $\operatorname{IgE}$ concentrations and the risk of positive food challenges in children and adolescents. J Allergy Clin Immunol. 1997;100:444-51. 
16. Nicolaou N, Poorafshar M, Murray C, Simpson A, Winell H, Kerry G, et al. Allergy or tolerance in children sensitized to peanut: prevalence and differentiation using component-resolved diagnostics. J Allergy Clin Immunol. 2010;125:191-7.

17. Sicherer SH, Wood RA. Advances in Diagnosing Peanut Allergy. J Allergy Clin Immunol Pract. 2013;1(1):1-13; quiz14.

18. Kim HY, Han Y, Kim K, Lee JY, Kim MJ, Ahn K, et al. Diagnostic Value of Specific IgE to Peanut and Ara h2 in Korean Children with Peanut Allergy. Allergy Asthma Immunol Res. 2016;8(2):156-60.

19. Ebisawa M, Moverare R, Sato S, Maruyama N, Borres MP, Komata T. Measurement of Ara h 1-, 2-, and 3-specific IgE antibodies is useful in diagnosis of peanut allergy in Japanese children. Pediatr Allergy Immunol. 2012;23:573-81

20. Lieberman JA, Glaumann S, Batelson S, Borres MP, Sampson HA, Nilsson C. The utility of peanut components in the diagnosis of IgE-mediated peanut allergy among distinct populations. J Allergy Clin Immunol Pract. 2013;1(1):75-82.

21. Ma S, Nie L, Li H, Wang R, Yin J. Component-Resolved Diagnosis of Peanut Allergy and Its Possible Origins of Sensitization in China. Int Arch Allergy Immunol. 2016;169:241-8

22. Lin YT, Wu CT, Cheng JH, Huang JL, Yeh KW. Patterns of sensitization to peanut allergen components in Taiwanese Preschool children. J Microbiol Immunol Infect. 2012;45:90-5.

23. Australian Society of Clinical Immunology and Allergy [Internet]. Australia: Australian Society of Clinical Immunology and Allergy (ASCIA) open peanut challenge protocol; c2011 [cited 2019 Mar 13]. Available from: http://allergy.org.au/images/stories/pospapers/ASCIA_food_allergy_ challenge_protocols_Sept_2011.pdf
24. Li PH, Rutkowski K, Kennard L, Sellaturay P, Watts TJ, Haque R, et al. Challenge-confirmed peanut allergy in older patients: Performance of skin tests, specific immunoglobulin E, and ara h 2. Ann Allergy Asthma Immunol. 2018;120(3):324-33.

25. Simms E, Foster G, Arias K. Larche M, Freitag T, Walker T, et al. Prediction of clinical peanut allergy status among children in Hamilton, Ontario using chart review data collected during 2012-2015. Allergy Asthma Clin Immunol. 2017;13:10.

26. Gray CL, Levin ME, du Toit G. Ethnic differences in peanut allergy patterns in South African children with atopic dermatitis. Pediatr Allergy Immunol. 2015;26(8):721-30.

27. Suratannon N, Ngamphaiboon J, Wongpiyabovorn J, Puripokai P, Chatchatee P. Component-resolved diagnostics for the evaluation of peanut allergy in a low-prevalence area. Pediatr Allergy Immunol. 2013. 24(7):665-70

28. Asarnoj A, Nilsson C, Lidholm J, Glaumann S, Ostblom E, Hedlin G, et al Peanut component Ara h 8 sensitization and tolerance to peanut. J Allergy Clin Immunol. 2012;130(2):468-72.

29. en.wikipedia.org [Internet]. California: Betula pendula; c2019[cited 2019 Mar 13]. Distribution and habitat; [about 2 screens]. Available from: https://en.wikipedia.org/wiki/Betula_pendula\#cite_note-GRIN-7

30. Asero R, Fernandez-Rivas M, Knulst AC, Bruijnzeel-Koomen CA Double-blind, placebo-controlled food challenge in adults in everyday clinical practice: a reappraisal of their limitations and real indications. Curr Opin Allergy Clin Immunol. 2009;9(4):379-85. 